\title{
Afferent loop syndrome caused by stenosis of jejunojejunal anastomosis of Roux-en-Y reconstruction
}

\author{
G. P. U. P. de Silva, M. S. E. Karunadasa, P. S. K. Madugalle, H. M. S. S. B. Rathnayake \\ Surgical Unit, Base Hospital Mahiyanganaya
}

Key words: Afferent loop; roux-en-Y; billroth II; exploratory laparotomy

\section{Introduction}

Afferent loop syndrome (ALS) is an infrequent complication originally described with Billroth II gastrojejunostomy. It is caused by mechanical obstruction owing to internal herniation, volvulus or narrowing of the anastomosis. Similarly, an obstruction of the biliopancreatic limb of Rouxen-Y reconstruction can rarely cause ALS. Early diagnosis and surgical intervention are crucial to decrease morbidity and mortality in acute ALS.

\section{Case presentation}

We present a case of a 32 years old woman presented with moderate to severe epigastric pain and non-bilious vomiting for the one-day duration. The pain was radiated to the back and slightly relieved with bending forward. She had opened bowel as usual. She has undergone gastrojejunostomy with a Roux-en-Y reconstruction 2 years ago due to stricture at the gastric antrum following corrosive ingestion. She described similar less severe episodes over the past year for which she used to take outpatient treatment. She was tachycardic but neither febrile nor icteric. Abdominal examination revealed epigastric tenderness with localized guarding without a positive Murphy's sign.

Laboratory data showed very high serum amylase and $C$ reactive protein with marginally elevated leucocyte count. Plain radiography showed a sentinel loop of the small intestine at the upper abdomen without features of pneumoperitoneum. Ultrasonography revealed features of acute pancreatitis, a close by aperistaltic loop of the small bowel and a normal biliary tree. Based on these findings, the patient was diagnosed and treated as acute pancreatitis. Despite the treatment, her condition was deteriorated and required intensive care after 48 hours of admission. Contrastenhanced CT abdomen was performed at the nearest centre

\footnotetext{
Correspondence: G. P. U. P. de Silva

E-mail: upulonline@gmail.com

Received: 11-06-2019 Accepted: 07-07-2019

(iD https://orcid.org/0000-0003-2508-3765 DOI: http://doi.org/10.4038/sljs.v37i2.8631
}

where the facilities were available. It revealed a dilated afferent loop and a concealed perforation. Emergency exploratory laparotomy was performed and detected a stenosed jejunojejunal anastomosis site (Figure 1) and grossly dilated afferent loop with a perforation at first part of the duodenum opening into the less sac. Perforation was sutured primarily with an omental patch. Stenosed Jejunojejunostomy was disconnected and new jejunojejunal anastomosis was created.

Despite the surgery, she continued to have a high output biliary drain through the surgical site drain. She was managed as for high output enterocutaneous fistula with sepsis control, total parenteral nutrition. The decision was made for the reexploration as there was no significant recovery. Reexploration revealed two further perforations at the first and second parts of the duodenum (Figure 2). Duodenojejunostomy was created at the larger perforation site with a loop of the jejunum and smaller perforation was repaired primarily after intra-operative hepatobiliary opinion. The patient expired on the tenth day after admission despite continued intensive care.

\section{Discussion}

ALS is a mechanical complication that occurs in $1 \%$ of the afferent loops of Billroth II gastrojejunostomy. It has been

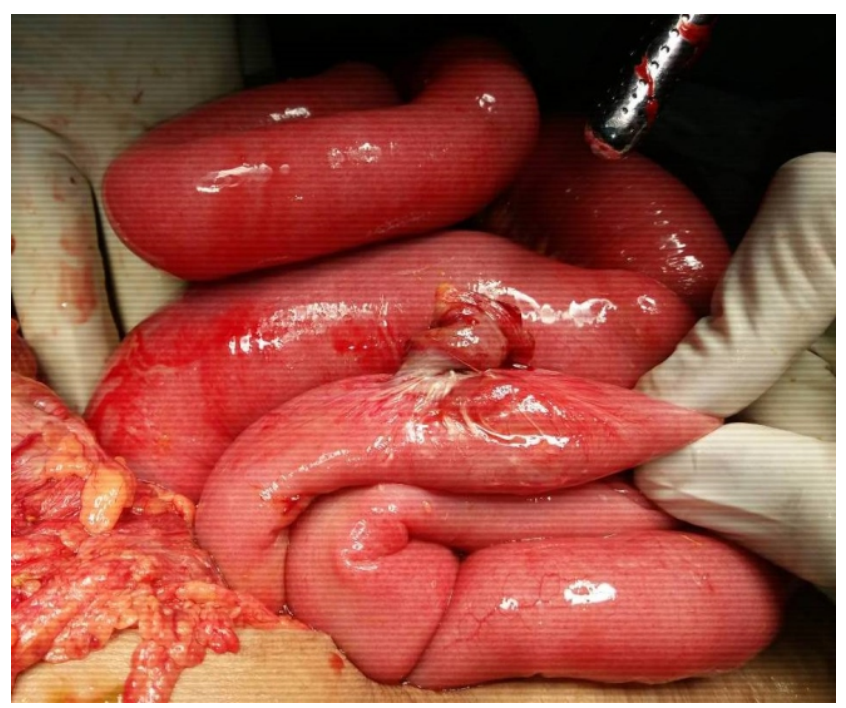

Figure 1. Stenosed jejunojejunal anastomosis 


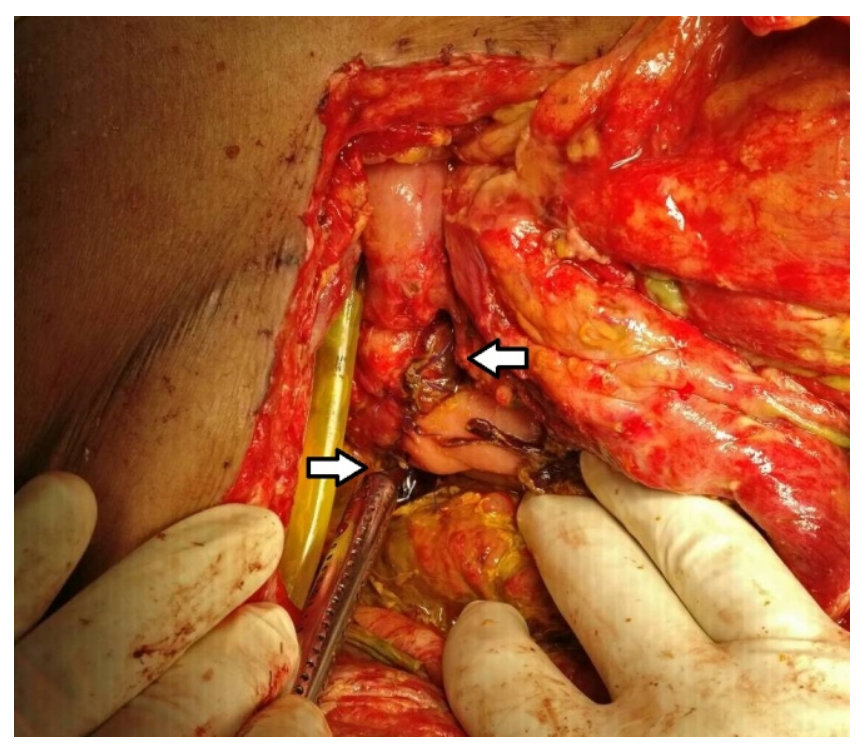

Figure 2. Duodenal perforations (marked with arrowheads) detected during re-exploration

reported similarly with mechanical obstruction of the biliopancreatic loop of the Roux-en-Y reconstruction as in our patient [1].

The afferent loop consists of the duodenum and proximal jejunum, lying upstream from the gastrojejunostomy/ jejunojejunostomy. This conveys about 11 of bile, pancreatic juice and proximal intestinal secretions to the jejunum per day under gastrointestinal hormonal influence. When there is a distal mechanical obstruction, accumulation of enteric secretions increases the intraluminal pressure resulting distension of the segment, if unrelieved can lead to ischemia, gangrene with subsequent perforation. In addition, the transmission of high luminal pressure may impair the sphincter mechanisms of biliopancreatic duct system resulting in ascending cholangitis and acute pancreatitis. Causes for the obstruction can be compression by postoperative adhesions, internal hernia, volvulus, enteroenteral intussusception, kinking or stenosis of jejunojejunostomy anastomosis, recurrent malignancy and rarely the enterolith or bezoars [2]. Our patient had a stenosis of the jejunojejunostomy which could be due to chronic ischemia caused by tension or malposition. Some studies have shown that longer, redundant and antecolic afferent limbs are more prone to ALS [3]. Even though the early diagnosis and intervention are critical to decreasing the mortality in acute ALS, the diagnosis is challenging due to its rarity and non-specific symptoms [3]. ALS can manifest as acute or chronic presentations. Acute ALS presents with complete obstruction of the loop as in our patient. Vomiting may not be profuse and non-bilious due to the complete obstruction of the loop. The chronic form is associated with partial obstruction and manifests as postprandial epigastric pain, projectile bilious vomiting and nutritional deficiencies. Acute pancreatitis or obstructive jaundice may coexist as described above making the diagnosis more challenging. Early use of CT imaging would have been ideal in our case, but again it is a challenge in a resource-poor setting. Treatment includes initial resuscitation and surgery for relieving of the cause for obstruction and reconstruction of the afferent limb in benign conditions. If the afferent loop is redundant, it needs to be shortened to $20-30 \mathrm{~cm}$ [3]. If the obstruction is of malignant in origin, the aim of treatment would be the palliation. Imageguided percutaneous drainage and endoscopic stent placement have been reported as palliative methods for malignant causes in specialized centres. [4]

\section{Conclusion}

Even though acute ALS is a rare complication, it must be considered as a differential diagnosis for acute abdomen in patients who have previously undergone Roux-en-Y reconstruction considering the necessity of early diagnosis and surgical intervention to reduce the mortality.

All authors disclose no conflict of interest. The study was conducted in accordance with the ethical standards of the relevant institutional or national ethics committee and the Helsinki Declaration of 1975, as revised in 2000 .

\section{References}

1. Aoki M, Saka M, Morita S, Fukagawa T, Katai H. Afferent Loop Obstruction After Distal Gastrectomy with Roux-en-Y Reconstruction. World J Surg. 2010;34(10):2389-92. https://doi.org/10.1007/s00268-010-0602-5.

2. Blouhos K, Boulas KA, Tsalis K, et al. Management of afferent loop obstruction: Reoperation or endoscopic and percutaneous interventions? World J Gastrointest Surg. 2015;7:190-5. https://doi.org/10.4240/wjgs.v7.i9.190.

3. Eagon J C, Miedema B W, Kelly K A, Postgastrectomy syndromes. Surg Clin Noth Am. 1992 Apr. 72(2):445-465.

4. Taunk P, Cosgrove N, Loren DE, Kowalski T, Siddiqui AA. Endoscopic ultrasound-guided gastroenterostomy using a lumenapposing self-expanding metal stent for decompression of afferent loop obstruction. Endoscopy. 2015;47 Suppl 1 UCTN:E395-E396. https://doi.org/10.1055/s-0034-1392564

\section{Learning Points:}

- Afferent loop syndrome (ALS) is a rare complication of Billroth II gastrojejunostomy and Roux-en-Y reconstruction.

- Diagnosis of acute ALS is challenging.

- Early surgical intervention is needed for acute ALS to reduce mortality. 